[18] Bujak, K., Wasilewski, J., Osadnik, T., Jonczyk, S., Kołodziejska, A., Gierlotka, M., Gąsior, M. (2015). The Prognostic Role of Red Blood Cell Distribution Width in Coronary Artery Disease: A Review of the Pathophysiology. Disease Markers, 2015, 1-12. doi: http://doi.org/10.1155/2015/824624

[19] Huang, Y.-L., Hu, Z.-D. (2016). Lower mean corpuscular hemoglobin concentration is associated with poorer outcomes in intensive care unit admitted patients with acute myocardial infarction. Annals of Translational Medicine, 4 (10), $190-190$. doi: http://doi.org/10.21037/atm.2016.03.42

[20] Turcato, G., Serafini, V., Dilda, A., Bovo, C., Caruso, B., Ricci, G., Lippi, G. (2016). Red blood cell distribution width independently predicts medium-term mortality and major adverse cardiac events after an acute coronary syndrome. Annals of Translational Medicine, 4 (13), 254. doi: http://doi.org/10.21037/atm.2016.06.35

\title{
BASIC FIBROBLAST GROWTH FACTOR AND ADIPONECTIN IN ADOLESCENCE WITH JUVENILE IDIOPATHIC ARTHRITIS TREATED WITH METHOTREXATE
}

\author{
Liudmyla Parkhomenko ${ }^{1}$ \\ parkhomenko.lk@gmail.com \\ Larysa Strashok ${ }^{1}$ \\ laspediatr1984@gmail.com \\ Olga Pavlova ${ }^{1}$ \\ ospavlova@mail.ua \\ ${ }^{1}$ Department Adolescence medicine \\ Kharkiv Medical academy of postgraduate education \\ 58 Amosova str., Kharkiv, Ukraine, 61176
}

\begin{abstract}
Methotrexate has been applied clinically for juvenile idiopathic arthritis (JIA) treatment for decades. It is recommended for use globally, according all modern guidelines. Despite the fact that fibrosis molecular mechanisms as well as methotrexate (MTX) elimination and fibrosis indexes were studied a lot there is still not enough information for adolescence. Adiponectin, fibroblast growth factor and fibrosis indexes in adolescents with JIA treated with methotrexate were studied in this work.

The aim was to study dynamics of molecular-cellular mechanisms activation of fibrotic processes development in the liver in adolescents with juvenile idiopathic arthritis treated with methotrexate.

Materials and methods: A total of 68 children with juvenile idiopathic arthritis, were enrolled in the study. 25 boys $(36.8 \%)$ and 43 girls $(63.2 \%)$ were examined. Children were divided into three groups in accordance with the methotrexate dose. The following data were analyzed: ESR ( $\mathrm{mm} /$ hour), C-reactive protein (mg/l), Hemolytic activity (CU), circulating immune complexes, (g/l), ALT (U/l), AST (U/l), Adiponectin (mcg/ml), BFGF (pg/ml), APRI index, FIB-4 Score.

Results: According to our results when patients start using MTX they have significantly positive effect. Therefore, when analyzing all parameters liver pathologies may occur before MTX use. When MTX used, its proinflammation and antifibrotic effects lead to normalization of all organs and systems, as well as joints and liver. Also, long-term MTX use can lead to adverse effects.

Conclusions: So, it is important to control possible liver disorders in adolescence treated with MTX. According to our study results we find out that there are decreasing of liver damage parameters in patients which started using MTX.

Keywords: Juvenile idiopathic arthritis, methotrexate, basic fibroblast growth factor, adiponectin.
\end{abstract}




\section{Introduction}

Juvenile idiopathic arthritis (JIA) is the most common chronic disease of childhood. One in 1,000 children is affected by the various subtypes of JIA. Methotrexate (MTX) is a cornerstone of therapy worldwide for JIA, yet there remains vast variability in drug dosing and administration, as well as unpredictable outcomes on the drug [1]. Methotrexate (4-amino-10-methylpholic acid) is commonly administered orally as a single weekly dose. MTX dosage is clearly regulated by modern treatment guidelines. After oral administration, MTX is absorbed in the proximal jejunum by the proton-coupled folate transporter, which transports reduced folates and MTX [2]. MTX maximum plasma concentrations are between 0,3 and 1,6 $\mu \mathrm{mol} / \mathrm{L}$, and occur at up to two hours after administration [3]. Also MTX can distribute to the synovial fluid with comparable levels to those found in plasma [4]. MTX side effects on the gastrointestinal tract are associated precisely with the antagonism of MTX and folate. Adding folic or folinic acid to therapy almost eliminates such side effects as nausea, vomiting, diarrhea, or stomatitis [3]. Manifestations of nausea and diarrhea are more often observed with oral administration of MTX [5]. Major elimination route of MTX is renal excretion constitutes. MTX is subject to first-pass metabolism in the liver and is converted to 7-hydroxymethotrexate, which is a major metabolite of MTX [6]. MTX in small proportion is excreted in the bile and some enterohepatic recycling also happens [3, 6, 7]. The plasma half-life of low dose MTX varies from 4.5 hours to 10 hours [4]. Hepatotoxicity is one of the main side effects of MTX in the JIA treatment. Slight increase in aminotransferases level, less commonly hepatic steatosis, fibrosis and cirrhosis are most common manifestations [8].

The precise mechanisms of MTX toxicity are still not clear. It was suggested that MTX hepatotoxicity may result from a depletion of hepatic folate stores and the accumulation of MTX polyglutamates in the liver [9]. However, folate supplementation has been associated with a reduced incidence of hepatic adverse effects (elevated transaminases) induced by MTX treatment [10].

MTX-related hepatic fibrosis may be mediated through an adenosine pathway. MTX was shown to enhance the release of adenosine from cultured hepatoma cells. Adenosine binds to the adenosine A2A receptor on hepatic stellate cells which is the principal fibrogenic cell type in the liver, and promotes collagen production [11, 12].

Moreover, MTX is known to interfere with the generation of methionine from homocysteine. Excess homocysteine can induce endoplasmic reticulum stress and promote fat accumulation in the liver. Homocysteine can also activate proinflammatory cytokines and hepatic stellate cells, leading to liver fibrosis [13]. MTX-induced hepatic damage may be related to reactive oxygen species generation. MTX was shown to cause oxidative tissue damage by increasing lipid peroxidation in the liver tissue and decreasing the level of antioxidant enzymes [14].

Fibrosis can cause impaired liver function due to changes in the tissue microenvironment, various growth factors synthesis, inflammatory cytokines functioning, a violation of the liver normal structure as a result of changes in the distribution and proportions of febrile collagen.

Despite the significant compensatory liver potential in childhood, it is important to prevent of steatosis and fibrosis development in the future. Adiponectin is the most common protein secreted by white adipose tissue. It circulates in the blood, and its concentration in serum is inversely proportional to fat mass. The wide distribution of adiponectin receptors in peripheral tissues and organs allows adiponectin to exert a pleiotropic effect on the general body metabolism. In addition to the well-known anti-diabetic, anti-atherogenic and anti-inflammatory properties, have been obtained data on the direct effect of adiponectin on non-alcoholic fatty liver disease development.

The study of the risk of developing fibrosis is possible including use of trigger factors for the development of fibrosis. Fibroblast growth factor (FGF) showed a potential effect on tissue repair and regeneration $[15,16]$. It was first identified as a protein capable of stimulating fibroblast proliferation, and it is now known that the group of these factors includes 22 members. FGFs perform multiple functions by binding and activating fibroblast growth factor receptors (FGFRs), and the main signalling pathway is FGFR stimulation leading tissue repair and regeneration [17, 18]. Fibroblast growth factor receptors 1, 2, and 4 are expressed in the liver, with FGFR4 exclusively in hepatocytes $[19,20]$. FGF1 and FGF2 are produced during hepatocyte replication and are mitogens for both endothelial and stellate liver cells [21]. 
Despite the fact that treatment with methotrexate JIA is the key, it makes sense to study the balance between its positive and negative effects, including finding out the risk of fibrosis.

The aim was to study dynamics of molecular-cellular mechanisms activation of fibrotic processes development in the liver in adolescents with juvenile idiopathic arthritis treated with MTX.

\section{Materials and methods}

The study group included JIA patients, a total of 68 children 25 boys $(36.8 \%)$ and 43 girls $(63.2 \%)$. The average age of patients was $13.3 \pm 0.3$ years. This study was carried out from 2017 to 2019.

Children were treated at the Cardiac-Rheumatology Department, State Institution "Institute for the Protection of Children and Adolescents' Health", Academy of Medical Sciences of Ukraine with Juvenile idiopathic arthritis. The diagnosis juvenile idiopathic arthritis was established in accordance with EULAR recommendations relevant at the time of the study. Treatment was carried out according to the same recommendations for all children. Informed consents' from all patients were obtained.

This work complies with ethical standards and it was approved by ethical committee of Kharkiv medical academy of postgraduate education from the $18^{\text {th }}$ of February 2020, protocol No. 1. This work was carried out according to the Helsinki Declaration.

Inclusion criteria's were boys and girls from 10 till 18 years old with JIA (with oligoarthritis and polyarthritis) treated with MTX or patients whom MTX were prescribed, but they did not received it yet on the moment of the study start.

Exclusion criteria's: children with chronic liver pathology, gastrointestinal, genetic and endocrinal disorders which occurred before JIA were diagnosed, children with uveitis. All examined patients had no viral hepatitis.

All adolescents underwent laboratory tests: clinical blood count, liver tests (AST (U/L), ALT (U/L)), acute phase indicator (C-reactive protein (mg/l)), Hemolytic activity (CU), circulating immune complexes, $(\mathrm{g} / \mathrm{l})$, Adiponectin $(\mathrm{mcg} / \mathrm{ml})$, BFGF $(\mathrm{pg} / \mathrm{ml})$. Abdominal organs ultrasound examination, joints ultrasound, and joints X-Ray were provided.

Level of adiponectin in the blood was determined by an enzyme-linked immunosorbent assay using the Human Adiponectin ELISA reagent kit, manufactured by BioVendor.

Determining the level of BFGF in the blood was carried out by an enzyme-linked immunosorbent assay using the Human bFGF ELISA reagent kit, manufactured by Elabscience.

Fibrosis indexes were calculated with Microsoft Excel program using standard formulas:

APRI index (AST to Platelet Ratio Index) was calculating using formula:

$$
\text { [(AST/ULN AST)×100]/Platelets (109/L)]. }
$$

FIB-4 score index (Fibrosis 4 Score) was calculated using following formula:

$$
(\text { Age } \times \text { AST }) /(\text { Platelets } \times(\operatorname{sqr}(\text { ALT })) .
$$

Adiponectin was studied to assess the risk of liver steatosis. Adiponectin was measured according to the instructions by ELISA. To assess the risk of liver fibrosis, the basic fibroblast growth factor (BFGF) was studied. We measured it according to the instructions by ELISA.

Children were divided into three groups according to the dose of methotrexate per $1 \mathrm{~m}^{2}$. The first group $(0(n=18))$ included children with JIA who had just been appointed MTX and they had not received it yet. In the second $(1(\mathrm{n}=21))$ were children who receive MTX less than $12.5 \mathrm{mg}$, third $(2(n=25))$ included ones who had dose more than $12.5 \mathrm{mg}$. The distribution of indicators was carried out in accordance with an average value of 12.5 .

Statistical analysis was carried out with software package Statistica 6.0. Before statistical processing, all data was checked for normal distribution. Normal distribution data were analyzed with a Pearson correlation method, a Spearman correlation was used for not 
normally distributed data relationships. One-way ANOVA was used to analyze differences between groups. LSD test was used to calculate the statistical significance. Differences were considered significant at $\mathrm{p}<0.05$.

\section{Results}

The proportions of gastrointestinal tract disorders associated in the study groups treated with MTX were as follows: nausea $-42.1 \%$, belching $-3.2 \%$, epigastric pain $-51.0 \%$ pain in the pyloroduodenal zone $-41.4 \%$, pain in the right hypochondrium $-37.7 \%$, abdominal pain $-21.7 \%$. Liver size increase during palpation was found in $73.8 \%$ of adolescents. The presented data cannot be the criteria for the diagnosis of fibrosis and steatosis. It seems to be of particular importance to be able to differentiate, based on biomarker analysis, between risk of steatosis and fibrosis development. Thus we analyzed biochemical parameters (Table 1) and their correlations.

Table 1

Laboratory parameters value in adolescents with JIA, taking into account the dose of MTX per $1 \mathrm{~m}^{2}$

\begin{tabular}{cccc}
\hline Grouping & $\mathbf{0}$ & $\mathbf{1}(<\mathbf{1 2 , 5})$ & $\mathbf{2}(>\mathbf{1 2 , 5})$ \\
\hline Indicator & $\mathrm{n}=18$ & $\mathrm{n}=21$ & $\mathrm{n}=25$ \\
ESR mm/hour & $5.33 \pm 1.45$ & $3.67 \pm 0.46$ & $6.04 \pm 1.26$ \\
Hemolytic activity, CU & $0.99 \pm 0.04$ & $0.94 \pm 0.1$ & $1 \pm 0.04$ \\
Circulating immune complexes, g/L & $1.17 \pm 0.062$ & $1.33 \pm 0.099$ & $1.21 \pm 0.045$ \\
ALT U/L & $20.9 \pm 1.97^{* 0,1}$ & $31.1 \pm 2.37$ & $25.8 \pm 1.79$ \\
AST U/L & $25.1 \pm 2.25^{* 0,1}$ & $32.4 \pm 2.39$ & $29.9 \pm 2.09$ \\
APRI index & $0.29 \pm 0.031$ & $0.36 \pm 0.04$ & $0.37 \pm 0.03$ \\
FIB-4 Score & $0.24 \pm 0.028$ & $0.30 \pm 0.023$ & $0.29 \pm 0.017$ \\
Adiponectin(mcg/ml) & $1.53 \pm 0.144$ & $1.51 \pm 0.08$ & $1.47 \pm 0.12$ \\
BFGF(pg/ml) & $6876.4 \pm 338.05$ & $7517.3 \pm 209.7$ & $7134.9 \pm 228.4$
\end{tabular}

Note: $* 0,1$ - the presence of a statistically significant difference between groups and figures is indicated; it is noted between which particular groups this difference is present $p<0,05$

In the same group correlation of BFGF with the degree of functional disorders of the joints $(\mathrm{r}=0.496, \mathrm{p}=0.036)$ were found. Thus, the more disorders in the joints, the more active are the processes associated with collagen metabolism as a result of JIA. An increase in the level of BFGF in the central bloodstream can lead to the activation of fibrotic processes in various organs and tissues, and especially in the liver. This may be a reflection of the increased risk of sclerotic processes in the liver.

Positive correlation between BFGF level and hepatomegaly according to ultrasound data $(\mathrm{r}=0.752, \mathrm{p}=0.014)$ may indicate both the spread of autoimmune inflammatory process to the liver and fibrosis.

Alterative processes in the liver associated with autoimmune inflammation are confirmed by the presence of a positive correlation between ALT and BFGF $(r=0.512, p=0.029)$. It is possible to assume that, on the one hand, hepatocyte necrosis takes place in the liver and at the same time proliferative processes occur in the vascular component of the organ stroma, which can enhance the acceleration of the fibrosis process. This is confirmed by the revealed positive correlation between the content of BFGF and the FIB-4 Score index $(r=0.546, p=0.019)$.

In this study, no correlation was found between platelets level, AST and BFGF, which indicates that their action is different, although not detected with statistics methods, which directed towards to fibrotic processes development, since the combination of these indicators in fibrosis index gives a positive correlation with BFGF. Inflammatory process activity also presented in this study with decrease hemolytic activity level $(\mathrm{r}=-0.878, \mathrm{p}=0.021)$ and an increase in BFGF level in the central bloodstream, which is typical for autoimmune disorders. 
A positive correlation of adiponectin and BFGF $(r=0.577, p=0.019)$ may reflect an increase in its anti-inflammatory effect upon activation of collagen synthesis, in addition, adiponectin inhibits the adhesion of monocytes to vascular endothelial cells.

On the other hand, an increase in adiponectin levels with increased fibrotic activity may be associated with a steatosis risk due to visceral adipose tissue development.

BFGF correlations in group 1 were presented with statistically significant dependence with CIC ( $r=0.476, p=0.039)$ which is associated with an increase of autoimmune process characteristic activity of the inflammation phase. In this group also, there were no statistically significant correlations with BFGF, which was noted in the previous group and negative correlations were found with AST $(r=-0.568, p=0.011)$ and the APRI index $(r=-0.647, p=0.002)$, which may indicate a rather low risk of fibrosis in this group. This may be due to the antifibrotic effect of MTX, both on the joints and on the liver.

Group 2 did not show statistically significant correlations. That is, the lack of correlations defined in the previous groups, possibly indicates effective treatment, a decrease in the activity of inflammation and fibrosing.

\section{Discussion}

Our findings showed that although a relatively large proportion of patients with JIA reported symptoms of nausea and epigastric pain, relatively few of these patients could in fact be diagnosed with steathosis or fibrosis. A large proportion of patients with JIA failed to achieve predicted values for the parameters of abnormal liver tests values. These findings are consistent with those of large population studies which suggest that taking folic acid reduces the negative effects of MTX when taken orally. These findings are consistent with those of large population studies [3].

Despite the fact that fibrosis molecular mechanisms as well as MTX elimination and fibrosis indexes were studied a lot there is still not enough information for adolescence. According to our results when patients start using MTX they have significantly positive effect. Therefore, when analyzing all parameters liver pathologies may occur before MTX use. When MTX started its proinflammation and antifibrotic effects lead to normalization of all organs and systems, as well as joints and liver. Also, long-term MTX using can lead to MTX hepatotoxicity caused by MTX polyglutamates accumulation in the liver [9] or folate supplementation [10] which was reported by others.

So, it is important to control possible liver disorders in adolescence treated with MTX. According to our study results we find out that there are decreasing of liver damage parameters in patients which started using MTX.

The coexistence of antifibrotic effects of MTX, its gastric side effects and risk of liver steatosis and fibrosis development in patients with JIA requires further studies aiming to determine the mutual relationship of these phenomena and their association with liver risk. MTX-related hepatic fibrosis through an adenosine pathway was reported [11]. Homocysteine can also activate proinflammatory cytokines and hepatic stellate cells, leading to liver fibrosis [13]. MTX-induced hepatic damage may be related to reactive oxygen species generation [14].

To apply this knowledge in the daily practice of a doctor further studies are necessary. BFGF may be a valuable complement to liver tests. Additionally, it can help identify abnormal liver functioning, which may be associated with the reported symptoms.

\section{Study limitations}

We are aware that the small sample size is a limitation of the study. Despite the fact that liver biopsy remains diagnostic standard it is invasive method and cannot be used to perform population screening and assess the course of the disease in patients with diagnosed steatosis or fibrosis. Therefore, liver biopsy was not performed, and we cannot definitively exclude liver fibrosis and steatosis. However, morphological study cannot be provided in pediatrics as a screening method. Our study comprised mostly adolescence with JIA treated with MTX without any other serious comorbidities and our results should not be extrapolated to the general population. On the other hand, the strength of our results is that we recruited adolescences with JIA without other serious chronic diseases, with different dose of MTX. 


\section{Prospects for further research}

In the future, it is planned to analyze the dependence of BFGF in patients with JIA with other parameters. It is also planned to study the correlation of the degree of differentiation of BFGF with hepatocyte grows factor. BFGF data analyzing with routine laboratory and instrumental studies is appropriate for the timely determination of the risks of developing irreversible pathological changes in the liver during JIA treatment with MTX.

\section{Conclusions}

In patients with JIA, the activity of systemic inflammation increases even before the start of treatment, as evidenced by a statistically significant correlation of BFGF level in group of patients who did not receive MTX. This indicates a close relationship between the intensity of the inflammatory process and collagen synthesis activation, which can further provoke liver fibrosis.

In patients who started receive MTX alterative processes in the liver associated with autoimmune inflammation can be reduced firstly according to obtained data. Adequate treatment of MTX in accordance with modern guidelines reduces the risk of fibrosis and the activity of the inflammatory process.

\section{Conflict of interests}

The authors declare that they have no conflicts of interest.

\section{References}

[1] Smolen, J. S., Landewé, R., Bijlsma, J., Burmester, G., Chatzidionysiou, K., Dougados, M. et. al. (2017). EULAR recommendations for the management of rheumatoid arthritis with synthetic and biological disease-modifying antirheumatic drugs: 2016 update. Annals of the Rheumatic Diseases, 76 (6), 960-977. doi: http://doi.org/10.1136/annrheumdis-2016-210715

[2] Desmoulin, S. K., Hou, Z., Gangjee, A., Matherly, L. H. (2012). The human proton-coupled folate transporter. Cancer Biology \& Therapy, 13 (14), 1355-1373. doi: http://doi.org/10.4161/cbt.22020

[3] Grim, J., Chládek, J., Martínková, J. (2003). Pharmacokinetics and Pharmacodynamics of Methotrexate in Non-Neoplastic Diseases. Clinical Pharmacokinetics, 42 (2), 139-151. doi: http://doi.org/10.2165/00003088-200342020-00003

[4] Herman, R. A., Veng-Pedersen, P., Hoffman, J., Koehnke, R., Furst, D. E. (1989). Pharmacokinetics of Low-Dose Methotrexate in Rheumatoid Arthritis Patients. Journal of Pharmaceutical Sciences, 78 (2), 165-171. doi: http://doi.org/10.1002/ jps. 2600780219

[5] Goodman, S. M., Cronstein, B. N., Bykerk, V. P. (2015). Outcomes Related to Methotrexate Dose and Route of Administration in Patients with Rheumatoid Arthritis: A Systematic Literature Review. Clinical and Experimental Rheumatology, 33, 272-278.

[6] Seideman, P., Beck, O., Eksborg, S., Wennberg, M. (1993). The pharmacokinetics of methotrexate and its 7-hydroxy metabolite in patients with rheumatoid arthritis. British Journal of Clinical Pharmacology, 35 (4), 409-412. doi: http://doi.org/ 10.1111/j.1365-2125.1993.tb04158.x

[7] Inoue, K., Yuasa, H. (2014). Molecular Basis for Pharmacokinetics and Pharmacodynamics of Methotrexate in Rheumatoid Arthritis Therapy. Drug Metabolism and Pharmacokinetics, 29 (1), 12-19. doi: http://doi.org/10.2133/dmpk.dmpk-13-rv-119

[8] Conway, R., Carey, J. J. (2017). Risk of liver disease in methotrexate treated patients. World Journal of Hepatology, 9 (26), 1092-1100. doi: http://doi.org/10.4254/wjh.v9.i26.1092

[9] Kremer, J. M., Galivan, J., Streckfuss, A., Kamen, B. (1986). Methotrexate metabolism analysis in blood and liver of rheumatoid arthritis patients: Association with hepatic folate deficiency and formation of polyglutamates. Arthritis \& Rheumatism, 29 (7), 832-835. doi: http://doi.org/10.1002/art.1780290703

[10] Prey, S., Paul, C. (2009). Effect of folic or folinic acid supplementation on methotrexate-associated safety and efficacy in inflammatory disease: a systematic review. British Journal of Dermatology, 160 (3), 622-628. doi: http://doi.org/10.1111/j.13652133.2008.08876.x

[11] Chan, E. S. L., Montesinos, M. C., Fernandez, P., Desai, A., Delano, D. L., Yee, H. et. al. (2006). Adenosine A2Areceptors play a role in the pathogenesis of hepatic cirrhosis. British Journal of Pharmacology, 148 (8), 1144-1155. doi: http://doi.org/10.1038/ sj.bjp.0706812

[12] Che, J., Chan, E. S. L., Cronstein, B. N. (2007). Adenosine A2A Receptor Occupancy Stimulates Collagen Expression by Hepatic Stellate Cells via Pathways Involving Protein Kinase A, Src, and Extracellular Signal-Regulated Kinases 1/2 Signaling 
Cascade or p38 Mitogen-Activated Protein Kinase Signaling Pathway. Molecular Pharmacology, 72 (6), 1626-1636. doi: http:// doi.org/10.1124/mol.107.038760

[13] Ortega-Alonso, A., Andrade, R. J. (2018). Chronic liver injury induced by drugs and toxins. Journal of Digestive Diseases, 19 (9), 514-521. doi: http://doi.org/10.1111/1751-2980.12612

[14] Vardi, N., Parlakpinar, H., Cetin, A., Erdogan, A., Cetin Ozturk, I. (2010). Protective Effect of $\beta$-Carotene on Methotrexate-Induced Oxidative Liver Damage. Toxicologic Pathology, 38 (4), 592-597. doi: http://doi.org/10.1177/0192623310367806

[15] Nunes, Q. M., Li, Y., Sun, C., Kinnunen, T. K., Fernig, D. G. (2016). Fibroblast growth factors as tissue repair and regeneration therapeutics. PeerJ, 4, e1535. doi: http://doi.org/10.7717/peerj.1535

[16] Maddaluno, L., Urwyler, C., Werner, S. (2017). Fibroblast growth factors: key players in regeneration and tissue repair. Development, 144 (22), 4047-4060. doi: http://doi.org/10.1242/dev.152587

[17] Tsai, S.-J., Chen, T.-M., Chen, Y.-H., Sun, Hs. (2019). Fibroblast growth factors: Potential novel targets for regenerative therapy of osteoarthritis. Chinese Journal of Physiology, 62 (1), 2. doi: http://doi.org/10.4103/cjp.cjp_11_19

[18] Ren, X., Zhao, M., Lash, B., Martino, M. M., Julier, Z. (2020). Growth Factor Engineering Strategies for Regenerative Medicine Applications. Frontiers in Bioengineering and Biotechnology, 7. doi: http://doi.org/10.3389/fbioe.2019.00469

[19] Kang, J., Hu, J., Karra, R., Dickson, A. L., Tornini, V. A., Nachtrab, G. et. al. (2016). Modulation of tissue repair by regeneration enhancer elements. Nature, 532 (7598), 201-206. doi: http://doi.org/10.1038/nature17644

[20] Hou, J., Kan, M., McKeehan, K., McBride, G., Adams, P., McKeehan, W. (1991). Fibroblast growth factor receptors from liver vary in three structural domains. Science, 251 (4994), 665-668. doi: http://doi.org/10.1126/science.1846977

[21] Kan, M., Huang, J. S., Mansson, P. E., Yasumitsu, H., Carr, B., McKeehan, W. L. (1989). Heparin-binding growth factor type 1 (acidic fibroblast growth factor): a potential biphasic autocrine and paracrine regulator of hepatocyte regeneration. Proceedings of the National Academy of Sciences, 86 (19), 7432-7436. doi: http://doi.org/10.1073/pnas.86.19.7432

Received date 27.05.2020

Accepted date 20.06.2020

Published date 31.07.2020
(C) The Author(s) 2020

This is an open access article under the CC BY license (http://creativecommons.org/licenses/by/4.0). 